\title{
SET OPERATOR DECOMPOSITION AND CONDITIONALLY TRANSLATION INVARIANT ELEMENTARY OPERATORS
}

\author{
G. J. F. BANON \\ Instituto Nacional de Pesquisas Espaciais, Divisão de Processamento de Imagens \\ CP 515 \\ 12 201-970, São José dos Campos, SP, Brazil \\ banon@dpi.inpe.br \\ J. BARRERA \\ Instituto de Matemática e Estatística, Universidade de São Paulo \\ CP IME 20570 \\ 01 498-970, São Paulo, SP, Brazil \\ jb@ime.usp.br
}

\begin{abstract}
In the first part, we recall the axiomatic definition of the elementary morphological operators (dilations, erosions, anti-dilations and anti-erosions) and their characterization in the case of Boolean lattices. This characterization is used to derive the set operator decompositions from the general decompositions of operators between complete lattices. In the second part, we define the notions of "conditionally translation invariant" (c.t.i.) and of "locally c.t.i." elementary operators. These operators are those usually implemented on digital computers. We show how any c.t.i. elementary operator can be decomposed in terms of locally ones.
\end{abstract}

Key words: set operator decomposition, conditionally translation invariant elementary operator, Boolean lattice.

\section{Introduction}

In Image Processing, to work with translation invariant (t.i.) operators we must assume that the image domain has a torus structure. Unfortunately, this assumption is not acceptable in many practical applications. For this reason, the linear operators or the morphological elementary operators commonly used in Image Processing, behave like t.i. operators only in the "central" area of the image domain.

In the first part of the paper, we recall the axiomatic definitions of the four classes of morphological elementary operators. When the domain or the range of these elementary operators is a Boolean lattice, we can characterize the pairs of elementary operators that form Galois connections. This characterization is useful for two reasons: on one hand, it gives the clue for the link between any operator and its left or right kernel, and, on the other hand, it can be used to characterize the elementary set operators in terms of structuring functions.

Based on theses results, we derive the set operator decompositions from the general operator decompositions introduced by Banon and Barrera (1993).

In the second part of the paper, we introduce the formal definition of the so-called conditionally translation invariant (c.t.i.) elementary operators. This definition corresponds to what is usually implemented on digital computers and it is based on the notion of c.t.i. structuring function. We present the collection of all the structuring elements that characterize the c.t.i. structuring functions.

Unfortunately, the c.t.i. elementary operators are not sufficient to represent any operator. For this reason, we introduce the notion of locally c.t.i. elementary operator and we 
give a constructive decomposition of any c.t.i. elementary operator. The decomposition of a c.t.i. dilation is given as an example to illustrate the theory.

\section{Axiomatic Definition and Characterization of the Morphological Elementary Operators}

Let $(\mathcal{L}, \leq)$, or simply $\mathscr{L}$, be a complete lattice (Birkhoff, 1967). We denote by $\vee, \wedge$ the union and intersection in $\mathcal{L}$. The dual complete lattice of $\mathcal{L},(\mathcal{L}, \geq)$, is denoted $\widetilde{L}$.

Let $\mathscr{L}_{1}$ and $\mathscr{L}_{2}$ be two complete lattices. The class $\mathscr{L}_{2}{ }^{\alpha_{1}}$ of the mappings $\psi$ from $\mathscr{L}_{1}$ to $\mathscr{L}_{2}$, called operators, equipped with the punctual ordering $\underset{\left(\mathscr{L}_{2}\right)}{\overleftarrow{L^{2}}}$

$$
\psi \underset{\left(\mathscr{L}_{2}\right)}{\leq} \psi^{\prime} \Leftrightarrow \psi(X) \underset{\mathscr{L}_{2}}{\leq} \psi^{\prime}(X)\left(X \in \mathscr{L}_{1}\right)
$$

is a complete lattice.

We define the morphological elementary operators axiomatically. The dilations (resp. erosions) from $\mathscr{L}_{1}$ to $\mathscr{L}_{2}$ are the operators in $\mathscr{L}_{2}^{\mathcal{L}_{1}}$ which commute with union (resp. intersection). The anti-dilations (resp. anti-erosions) from $\mathscr{L}_{1}$ to $\mathscr{L}_{2}$ are the dilations (resp. erosion) from $\mathscr{L}_{1}$ to $\widetilde{L}_{2}$ (Banon \& Barrera, 1993).

We will denote by $\Delta\left(\mathscr{L}_{1}, \mathscr{L}_{2}\right), \mathrm{E}\left(\mathscr{L}_{1}, \mathscr{L}_{2}\right), \Delta^{\mathrm{a}}\left(\mathscr{L}_{1}, \mathscr{L}_{2}\right)$ and $\mathrm{E}^{\mathrm{a}}\left(\mathscr{L}_{1}, \mathscr{L}_{2}\right)$, respectively, the class of dilations, erosions, anti-dilations and anti-erosions from $\mathscr{L}_{1}$ to $\mathscr{L}_{2}$. We know (Banon \& Barrera, 1993a) that these classes are complete lattices. Consequently, the Galois connections $(\alpha, \beta)$ between $\mathscr{L}_{1}$ and $\mathscr{L}_{2}$ (Birkhoff, 1967) form a complete lattice with respect the the partial ordering

$$
(\alpha, \beta) \leq\left(\alpha^{\prime}, \beta^{\prime}\right) \Leftrightarrow \alpha \underset{\left(\mathscr{L}_{2}\right)}{\underset{2}{<}} \alpha^{\prime} \text { and } \beta \underset{\left(\mathscr{L}_{1}\right)}{\leq} \beta^{\prime} .
$$

If $(\alpha, \beta)$ is a Galois connection, then $\beta$ is the companion operator of $\alpha$ and similarly $\alpha$ is the companion operator of $\beta$.

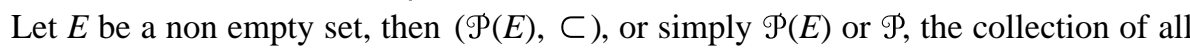
parts of $E$ equipped with the inclusion $\subset$, is a complete Boolean lattice. We denote by $X^{\mathrm{c}}$ the set complement of a subset $X$ of $E$.

Let $(\mathscr{L}, \leq)$ be a complete lattice. The set $\mathscr{L}^{E}$ of the functions $a$ from $E$ to $\mathscr{L}$ equipped with the punctual ordering $\underset{(\mathscr{L})}{\longrightarrow}$ is a complete lattice.

We now recall Corollary 4 of Achache (1982). A similar result (with $\mathcal{L}=\mathscr{P}(E)$ ) is in Serra (1988, Section 2.2).

Proposition 1 - The mapping $a \mapsto(\alpha, \beta)$ from the complete lattice $\mathcal{L}^{E}$ to the complete lattice of Galois connections between $\mathscr{L}$ and $(\mathscr{P}(E), \subset)$ defined by

$$
\alpha(X)=\left\{y \in E: X_{\mathfrak{L}}^{\leq} a(y)\right\}(X \in \mathcal{L}) \text { and } \beta(Y)=\bigwedge_{y \in Y} a(y)(Y \in \mathscr{P}(E))
$$

is a lattice isomorphism. Its inverse $(\alpha, \beta) \mapsto a$ is given by $a(y)=\beta(\{y\})(y \in E)$.

We know (Achache, 1982, Lemma 1) that $\alpha \in \Delta^{\mathrm{a}}(\mathcal{L}, \mathscr{P})$ and $\beta \in \Delta^{\mathrm{a}}(\mathscr{P}, \mathscr{L})$. From Proposition 1 we can derive the following proposition.

Proposition 2 - The mapping $a \mapsto(\alpha, \beta)$ from the complete lattice $\mathscr{L}^{E}$ to the complete lattice of Galois connections between $\mathcal{L}$ and $(\Phi(E), \supset)$ defined by 


$$
\alpha(X)=\{y \in E: X \underset{\mathcal{L}}{\leq} a(y)\}^{\mathrm{c}}(X \in \mathcal{L}) \text { and } \beta(Y)=\bigwedge_{y \in Y^{\mathrm{c}}} a(y)(Y \in \mathcal{P}(E))
$$

is a lattice isomorphism. Its inverse $(\alpha, \beta) \mapsto a$ is given by $a(y)=\beta\left(\{y\}^{c}\right)(y \in E)$.

In this case, $\alpha \in \Delta(\mathcal{L}, \mathscr{P})$ and $\beta \in \mathrm{E}(\mathscr{P}, \mathcal{L})$. In Propositions 1 and 2 the function $a$ is called the structuring function of the elementary operators $\alpha$ and $\beta$.

From Propositions 1 and 2 we can derive the elementary operators characterization given in Table 1 and the next corollary where $\mathscr{P}_{2}$ stands for $\mathscr{P}\left(E_{2}\right)$.

TABLE 1

Elementary operators characterization.

\begin{tabular}{|c|c|c|c|c|}
\hline $\begin{array}{l}\text { identifying } \\
\& \text { to }\end{array}$ & $\begin{array}{l}\text { in } \\
\text { Prop. }\end{array}$ & leads to & and & with \\
\hline$\tilde{L}_{1}$ & 1 & $\begin{array}{l}\alpha \in \mathrm{E}\left(\mathscr{L}_{1}, \mathscr{P}_{2}\right) \\
\beta \in \Delta\left(\mathscr{P}_{2}, \mathscr{L}_{1}\right)\end{array}$ & $\begin{array}{l}\alpha=\varepsilon_{a} \\
\beta=\delta_{a}\end{array}$ & $\begin{array}{c}\varepsilon_{a}(X)=\left\{y \in E_{2}: a(y) \leq X\right\} \\
\delta_{a}(Y)=\bigvee_{y \in Y} a(y)\end{array}$ \\
\hline $\mathscr{L}_{1}$ & 1 & $\begin{array}{l}\alpha \in \Delta^{\mathrm{a}}\left(\mathscr{L}_{1}, \mathscr{P}_{2}\right) \\
\beta \in \Delta^{\mathrm{a}}\left(\mathscr{P}_{2}, \mathscr{L}_{1}\right)\end{array}$ & $\begin{array}{l}\alpha={ }_{b} \delta^{\mathrm{a}} \\
\beta=\delta_{b}^{\mathrm{a}}\end{array}$ & $\begin{array}{c}{ }_{b} \delta^{\mathrm{a}}(X)=\left\{y \in E_{2}: X \leq b(y)\right\} \\
\delta_{b}^{\mathrm{a}}(Y)=\bigwedge_{y \in Y} b(y)\end{array}$ \\
\hline$\tilde{L}_{1}$ & 2 & $\begin{array}{l}\alpha \in \mathrm{E}^{\mathrm{a}}\left(\mathcal{L}_{1}, \mathscr{P}_{2}\right) \\
\beta \in \mathrm{E}^{\mathrm{a}}\left(\mathscr{P}_{2}, \mathscr{L}_{1}\right)\end{array}$ & $\begin{array}{l}\alpha=\varepsilon_{a}^{\mathrm{a}} \\
\beta={ }_{a} \varepsilon^{\mathrm{a}}\end{array}$ & $\begin{array}{c}\varepsilon_{a}^{\mathrm{a}}(X)=\left\{y \in E_{2}: a(y) \leq X\right\}^{\mathrm{c}} \\
{ }_{a} \varepsilon^{\mathrm{a}}(Y)=\bigvee_{y \in Y^{\mathrm{c}}} a(y)\end{array}$ \\
\hline $\mathscr{L}_{1}$ & 2 & $\begin{array}{l}\alpha \in \Delta\left(\mathscr{L}_{1}, \mathscr{P}_{2}\right) \\
\beta \in \mathrm{E}\left(\mathscr{P}_{2}, \mathscr{L}_{1}\right)\end{array}$ & $\begin{array}{l}\alpha={ }_{b} \delta \\
\beta={ }_{b} \varepsilon\end{array}$ & $\begin{array}{c}{ }_{b} \delta(X)=\left\{y \in E_{2}: X \leq b(y)\right\}^{\mathrm{c}} \\
{ }_{b} \varepsilon(Y)=\bigwedge_{y \in Y^{\mathrm{c}}} b(y)\end{array}$ \\
\hline
\end{tabular}

Corollary 3 - Let $a$ and $b$ be two functions from $E_{2}$ to $\mathscr{L}_{1}$. Then we have:

(1) if $a$ is the structuring function of $\delta \in \Delta\left(\mathscr{P}_{2}, \mathcal{L}_{1}\right)$ (i.e., $a(y)=\delta(\{y\})\left(y \in E_{2}\right)$ ) then its companion erosion is $\varepsilon_{a}$;

(2) if $b$ is the structuring function of $\delta^{\mathrm{a}} \in \Delta^{\mathrm{a}}\left(\mathscr{\Phi}_{2}, \mathcal{L}_{1}\right)$ (i.e., $\left.b(y)=\delta^{\mathrm{a}}(\{y\})\left(y \in E_{2}\right)\right)$ then its companion anti-dilation is ${ }_{b} \delta^{\text {a }}$;

(3) if $a$ is the structuring function of $\varepsilon^{\mathrm{a}} \in \mathrm{E}^{\mathrm{a}}\left(\mathscr{P}_{2}, \mathcal{L}_{1}\right)$ (i.e., $\left.a(y)=\varepsilon^{\mathrm{a}}\left(\{y\}^{\mathrm{c}}\right)\left(y \in E_{2}\right)\right)$ then its companion anti-erosion is $\varepsilon_{a}^{\text {a }}$;

(4) if $b$ is the structuring function of $\varepsilon \in \mathrm{E}\left(\mathscr{P}_{2}, \mathcal{L}_{1}\right)$ (i.e., $\left.b(y)=\varepsilon\left(\{y\}^{\mathrm{c}}\right)\left(y \in E_{2}\right)\right)$ then its companion dilation is ${ }_{b} \delta$.

\section{Operator Decomposition in terms of Elementary Operators}

In order to specialize to set operators the general decomposition theorem (Banon \& Barrera, 1993) we need to derive one more corollary from Proposition 1 . In this section $\mathscr{P}_{1}$ and $\mathscr{P}_{2}$ stands, respectively, for $\mathscr{P}\left(E_{1}\right)$ and $\mathscr{P}\left(E_{2}\right)$. Let $\psi$ be an operator from $\mathscr{P}_{1}$ to $\mathcal{P}_{2}$. We recall (Banon \& Barrera, 1993) that the mappings $\cdot \mathscr{K}(\psi)$ and $\mathscr{K} \cdot(\psi)$ from $\mathscr{P}_{2}$ to $\mathscr{P}\left(\mathscr{P}_{1}\right)$ given by, for any $Y \in \mathscr{P}_{2}$,

$$
\text { - } \mathscr{F}(\psi)(Y)=\left\{X \in \mathscr{P}_{1}: Y \subset \psi(X)\right\} \text { and } \mathscr{K} \cdot(\psi)(Y)=\left\{X \in \mathscr{P}_{1}: \psi(X) \subset Y\right\}
$$

are called, respectively, the left and right kernel of $\psi$. 
Corollary 4 - The left and right kernel of a set operator from $\mathscr{P}_{1}$ to $\mathscr{P}_{2}$ are, respectively, an anti-dilation and an erosion from $\mathscr{\Phi}_{2}$ to $\mathscr{P}\left(\Phi_{1}\right)$.

Proof - For the left kernel, the result follows from Proposition 1 by identifying $E, \mathcal{L}, a$ and $\alpha$ to, respectively, $\mathscr{P}_{1}, \Phi_{2}, \psi$ and $\cdot \mathscr{T}_{6}(\psi)$. For the right kernel, the result follows from Proposition 1 by identifying $E, \mathcal{L}, a$ and $\alpha$ to, respectively, $\mathscr{P}_{1}, \widetilde{\Phi}_{2}, \psi$ and $\mathscr{G} \cdot(\psi)$.

Let $\alpha, \beta \in \mathscr{\Phi}_{1}^{\Phi_{2}}$ and let $[\alpha, \beta]$ be the interval function from $\mathscr{P}_{2}$ to $\mathscr{P}\left(\mathscr{P}_{1}\right)$ with extremities $\alpha$ and $\beta$ (Banon \& Barrera, 1993).

Lemma 5 - Let $\psi$ be a set operator from $\mathscr{P}_{1}$ to $\mathscr{P}_{2}$.

(1) If $\alpha \in \Delta\left(\Phi_{2}, \mathscr{P}_{1}\right)$ and $\beta \in \Delta^{\mathrm{a}}\left(\mathscr{\Phi}_{2}, \mathscr{P}_{1}\right)$ then, for any $Y \in \mathscr{P}_{2}$,

$$
[\alpha, \beta](\{y\}) \subset \cdot \mathscr{K}_{0}(\psi)(\{y\})(y \in Y) \Rightarrow[\alpha, \beta](Y) \subset \cdot \mathscr{K}_{\mathcal{L}}(\psi)(Y) .
$$

(2) If $\alpha \in \mathrm{E}^{\mathrm{a}}\left(\mathscr{\Phi}_{2}, \mathscr{\Phi}_{1}\right)$ and $\beta \in \mathrm{E}\left(\mathscr{\Phi}_{2}, \mathscr{\Phi}_{1}\right)$ then, for any $Y \in \mathscr{P}_{2}$,

$$
[\alpha, \beta]\left(\{y\}^{c}\right) \subset \mathscr{K} \cdot(\psi)\left(\{y\}^{c}\right)\left(y \in Y^{c}\right) \Rightarrow[\alpha, \beta](Y) \subset \mathscr{K} \cdot(\psi)(Y) .
$$

Proof - Let us prove part (1). For any $Y \in \mathscr{P}_{2}$,

$$
\begin{array}{rlr}
\text { TRUE } & \Leftrightarrow[\alpha, \beta](Y) \subset[\alpha, \beta](\{y\})(y \in Y) & (\alpha \text { is isotone and } \beta \text { is antitone) } \\
& \Leftrightarrow[\alpha, \beta](Y) \subset \bigcap_{y \in Y}[\alpha, \beta](\{y\}) & \text { (property of the intersection) } \\
& \Rightarrow[\alpha, \beta](Y) \subset \bigcap_{y \in Y} \cdot \mathscr{G}_{\mathcal{G}}(\psi)(\{y\}) & ([\alpha, \beta](\{y\}) \subset \cdot \mathscr{G}(\psi)(\{y\})(y \in Y)) \\
& \Leftrightarrow[\alpha, \beta](Y) \subset \cdot \mathscr{G}_{\mathscr{G}}(\psi)\left(\bigcup_{y \in Y}\{y\}\right) & \text { (by Corollary 4, } \cdot \mathscr{G}_{\mathscr{G}}(\psi) \text { is an anti-dilation) } \\
& \Leftrightarrow[\alpha, \beta](Y) \subset \cdot \mathscr{G}_{\mathscr{G}}(\psi)(Y) . & (Y \text { representation by singletons) }
\end{array}
$$

The proof of part (2) is similar to the proof of part (1).

Let $\mathrm{AB}$ be the set defined by

$\mathrm{AB}=\left\{(a, b) \in \mathscr{P}_{1}{ }^{E_{2}} \times \mathscr{P}_{1}{ }^{E_{2}}: \forall y \in E_{2}, \quad(a(y) \subset b(y))\right.$ or $\left(a(y)=E_{1}\right.$ and $\left.\left.b(y)=\emptyset\right)\right\}$

Let $a, b \in \mathscr{P}_{1}^{E_{2}}$. We denote by $[a, b]$ the interval function from $E_{2}$ to $\mathscr{P}\left(\mathscr{P}_{1}\right)$ with extremities $a$ and $b$. We denote the punctual ordering $\underset{(\subseteq)}{\subseteq}$ on $\mathscr{P}\left(\mathscr{P}_{1}\right)^{E_{2}}$ simply $\leq$.

Theorem 6 - Any operator $\psi \in \mathscr{P}_{2}{ }^{\Phi_{1}}$ can be decomposed in terms of a set of sup-generating or inf-generating operators and the constructive decompositions are

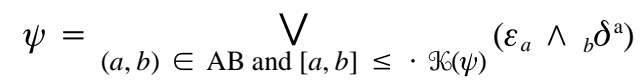

where $\cdot \mathscr{T}_{0}(\psi)(y)=\left\{X \in \mathscr{P}_{1}: y \in \psi(X)\right\}$, for any $y \in E_{2}$, and

$$
\psi=\bigwedge_{(a, b) \in \mathrm{AB} \text { and }[a, b] \leq \mathscr{F}_{6} \cdot(\psi)}\left(\varepsilon_{a}^{\mathrm{a}}{ }_{a} \vee_{b} \delta\right)
$$

where $\mathscr{T}_{\mathscr{C}} \cdot(\psi)(y)=\left\{X \in \mathscr{P}_{1}: y \notin \psi(X)\right\}$, for any $y \in E_{2}$.

Proof - We can make a direct proof or, as we do below, derive the result from the general decomposition theorem of Banon \& Barrera (1993). For any $\psi$ from $\mathscr{P}_{1}$ to $\Phi_{2}$, 


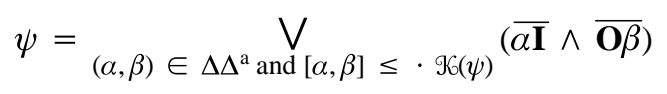

(Theorem 6.1 of Banon \& Barrera (1993a) or Theorem 1 of Banon \& Barrera (1993b))

$$
\begin{array}{ll}
=\bigvee_{(\alpha, \beta) \in \Delta \Delta^{\mathrm{a}} \text { and }\left([\alpha, \beta](y) \subset \cdot \mathscr{F}_{\mathcal{L}}(\psi)(\{y\})\left(y \in E_{2}\right)\right)}(\overline{\alpha \mathbf{I}} \wedge \overline{\mathbf{O} \beta}) & \text { (Lemma 5) } \\
=\bigvee_{(a, b) \in \mathrm{AB} \text { and }[a, b] \leq \cdot \mathscr{F}_{\mathcal{C}}(\psi)}\left(\varepsilon_{a} \wedge{ }_{b} \delta^{\mathrm{a}}\right) . & \text { (Corollary 3) }
\end{array}
$$

The proof of the second decomposition is similar to the proof of the first one.

A direct proof of a similar result is given in Banon \& Barrera (1990).

The sup-generating and inf-generating operators of Theorem 6 are, respectively, the operators $\varepsilon_{a} \wedge{ }_{b} \delta^{\mathrm{a}}$ and $\varepsilon_{a}^{\mathrm{a}}{ }_{a} \vee{ }_{b} \delta$, where $a$ and $b$ are functions from $E_{2}$ to $\mathscr{P}_{1}$.

\section{Conditionally Translation Invariant Elementary Operators}

Let $\left(\mathbf{Z}^{2},+\right)$ be the set of ordered pairs of integers equipped with the usual addition. Let $u$ be a point of $\mathbf{Z}^{2}$, we denote by $B+u$ the translate by $u$ of a subset $B$ of $\mathbf{Z}^{2}$ and by $B^{\mathrm{t}}$ its transpose (Banon \& Barrera 1991). From now on, we assume that the sets $E_{1}$ and $E_{2}$ of Section 3 are subsets of $\mathbf{Z}^{2}$ (for example "rectangles").

A function $b$ from $E_{2}$ to $\mathscr{P}_{1}$ is conditionally translation invariant or a ct-function iff

$$
\exists B \in \mathcal{\Phi}\left(\mathbf{Z}^{2}\right), \forall y \in E_{2}, b(y)=(B+y) \cap E_{1} .
$$

Let us consider the following subcollection $\mathscr{B}_{E_{1}, E_{2}}$, or simply $\mathscr{B}$, of $\mathscr{P}\left(\mathbf{Z}^{2}\right)$

$$
\mathscr{B}=\left\{B \in \mathscr{P}\left(\mathbf{Z}^{2}\right): \forall b \in B, \exists u \in E_{2}, b+u \in E_{1}\right\} .
$$

Let $\oplus$ denote the Minkowski addition on $\mathscr{P}\left(\mathbf{Z}^{2}\right)$ (Hadwiger, 1950). We observe that $\mathscr{B}$ is an ideal and a complete sublattice of $\mathscr{P}\left(\mathbf{Z}^{2}\right)$, its greatest element is $E_{1} \oplus E_{2}{ }^{\mathrm{t}}$ and $\mathscr{B}=\mathscr{P}\left(E_{1} \oplus E_{2}{ }^{t}\right)$. Figure 1 shows an element $B$ of $\mathscr{B}$ generated by two rectangles.

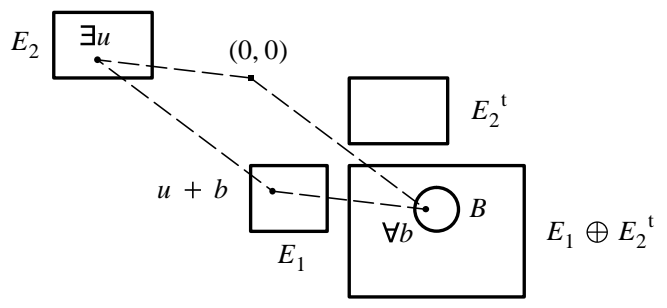

Fig. 1 - An element of an ideal generated by two rectangles.

The next proposition characterizes the ct-functions in $\mathscr{P}_{1}^{E_{2}}$ in terms of subsets of $E_{1} \oplus E_{2}{ }^{\mathrm{t}}$.

Proposition 7 - The mapping $B \mapsto b_{B}$ from $\mathscr{B}$ to the set of ct-functions in $\mathscr{P}_{1}^{E_{2}}$ given by

$$
b_{B}(y)=(B+y) \cap E_{1} \quad\left(y \in E_{2}\right)
$$

is a bijection. Its inverse $b \mapsto B_{b}$ is given by $B_{b}=\bigcup_{y \in E_{2}}(b(y)-y)$.

Proof - (1) For any $B \in \mathscr{B}, b_{B}$ is by construction a ct-function. 
(2) For any ct-function $b$ from $E_{2}$ to $\mathscr{P}\left(E_{1}\right)$

$$
\begin{array}{rrr}
B_{b} & =\bigcup_{y \in E_{2}}(b(y)-y) & \text { (definition of } \left.B_{b}\right) \\
& \subset \bigcup_{y \in E_{2}}\left(E_{1}-y\right) & \left(b(y) \subset E_{1}\right) \\
& =E_{1} \oplus E_{2}{ }^{\mathrm{t}}, & \text { (definition of } \oplus \text { ) }
\end{array}
$$

That is, $B_{b} \in \mathscr{B}$.

(3) Let us prove that $B \mapsto b_{B}$ is "one to one". On one hand, for any $B \in \mathscr{B}$,

TRUE $\Leftrightarrow(B+y) \cap E_{1} \subset(B+y) \quad\left(y \in E_{2}\right)$

$$
\begin{aligned}
& \Leftrightarrow\left((B+y) \cap E_{1}\right)-y \subset B \quad\left(y \in E_{2}\right) \\
& \Leftrightarrow \bigcup_{y \in E_{2}}\left(\left((B+y) \cap E_{1}\right)-y\right) \subset B \\
& \Leftrightarrow B_{b_{B}} \subset B .
\end{aligned}
$$

On the other hand, for any $B \in \mathscr{B}$ and $b \in \mathbf{Z}^{2}$,

$b \in B \Rightarrow b \in B$ and $\exists y \in E_{2}, b+y \in E_{1}$

$$
\begin{aligned}
& \Rightarrow \exists y \in E_{2}, b+y \in B+y \text { and } y+b \in E_{1} \\
& \Leftrightarrow \exists y \in E_{2}, b+y \in(B+y) \cap E_{1} \\
& \Leftrightarrow \exists y \in E_{2}, b \in\left((B+y) \cap E_{1}\right)-y \\
& \Leftrightarrow b \in \bigcup_{y \in E_{2}}\left(\left((B+y) \cap E_{1}\right)-y\right) \\
& \Leftrightarrow b \in B_{b_{B}} .
\end{aligned}
$$

(property of the intersection) (translation is isotone)

(property of the union)

(definitions of $b_{B}$ and $B_{b}$ )

(definition of $\mathscr{B}$ )

(property of the translation) (definition of the intersection) (translation is isotone)

(definition of the union)

(definitions of $b_{B}$ and $B_{b}$ )

That is, for any $B \stackrel{B}{\in}, B \subset B_{b_{B}}$. Therefore, $B \mapsto b_{B}$ is "one to one".

(4) Let us prove that $B \mapsto b_{B}$ is "onto". On one hand, for any ct-function $b$ in $\mathscr{P}_{1}^{E_{2}}$ and $y \in E_{2}$,

$$
\begin{aligned}
b_{B_{b}}(y) & =\left(\left(\bigcup_{v \in E_{2}}(b(v)-v)\right)+y\right) \cap E_{1} & \text { (definitions of } \left.B_{b} \text { and } b_{B}\right) \\
& \supset b(y) \cap E_{1} & (v=y \text { e property of the union) } \\
& =b(y) . & \left(b(y) \subset E_{1}\right)
\end{aligned}
$$

On the other hand, for any ct-function $b$ in $\mathscr{P}_{1}^{E_{2}}$ and $y \in E_{2}$,

$$
\begin{array}{rlr}
\text { TRUE } & \left.\Leftrightarrow\left(\left(\bigcup_{v \in E_{2}}(b(v)-v)\right)+y\right) \cap E_{1} \subset\left(\bigcup_{v \in E_{2}}(b(v)-v)\right)+y \quad \text { (property of } \cap\right) \\
& \left.\Leftrightarrow\left(\left(\left(\bigcup_{v \in E_{2}}(b(v)-v)\right)+y\right) \cap E_{1} \subset(b(v)-v)+y\right)\left(v \in E_{2}\right) \quad \text { (prop. of } \bigcup\right) \\
& \Leftrightarrow\left(b_{B_{b}}(y) \subset(b(v)-v)+y\right)\left(v \in E_{2}\right) & \text { (definitions of } \left.B_{b} \text { and } b_{B}\right) \\
& \Rightarrow b_{B_{b}}(y) \subset b(v) . & (v=y)
\end{array}
$$

Therefore, $B \mapsto b_{B}$ is "onto".

We say that an elementary operator from $\mathscr{P}\left(E_{1}\right)$ to $\mathscr{P}\left(E_{2}\right)$ is conditionally translation invariant (c.t.i.) iff its structuring function from $E_{2}$ to $\mathscr{P}\left(E_{1}\right)$ is a ct-function. For any $B \in \Re$, we denote by $\alpha_{B}$ (or ${ }_{B} \alpha$ ) the c.t.i. elementary operator which has the structuring function $b_{B}$. In particular, we have $\varepsilon_{B}(X)=\left(\left(X \cup E_{1}{ }^{\mathrm{c}}\right) \ominus B\right) \cap E_{2}\left(X \in \mathscr{P}\left(E_{1}\right)\right)$ and $\delta_{B}(Y)=(Y \oplus B) \cap E_{1}\left(Y \in \mathcal{P}\left(E_{2}\right)\right)$ where $\ominus$ is the Minkowski subtraction on $\mathscr{\Phi}\left(\mathbf{Z}^{2}\right)$ (Hadwiger, 1950). 


\section{Locally c.t.i. Elementary Operators}

A function $b$ from $E_{2}$ to $\mathscr{P}_{1}$ is said to be a locally ct-function iff there exist a subset $M$ of $E_{2}$, called mask, and a ct-function $b^{\prime}$ from $E_{2}$ to $\mathscr{P}_{1}$, such that

$$
b(y)=\left\{\begin{array}{ll}
b^{\prime}(y) & \text { if } y \in M \\
\emptyset & \text { otherwise }
\end{array} \quad\left(y \in E_{2}\right) .\right.
$$

We will now give a constructive function decomposition in terms of locally ct-function. Let $b$ be a function from $E_{2}$ to $\mathscr{P}_{1}$. We define the binary relation $\mathscr{R}_{b}$ on $E_{2}$ by

$$
y_{1} \mathscr{\Re}_{b} y_{2} \Leftrightarrow \exists B \in \mathscr{B},\left(B+y_{1}\right) \cap E_{1}=b\left(y_{1}\right) \text { and }\left(B+y_{2}\right) \cap E_{1}=b\left(y_{2}\right) \text {. }
$$

The relation $\mathscr{P}_{b}$ is an equivalence relation. We denote by $E_{2} / \mathscr{R}_{b}$ the resulting partition of $E_{2}$.

Proposition 8 - Any function $b$ from $E_{2}$ to $\mathscr{P}_{1}$ can be decomposed in terms of locally ctfunctions and the constructive decomposition is

$$
b=\bigvee_{M \in E_{2} / \mathscr{R}_{b}} b_{B_{b / M^{M}}}
$$

where, for any $M \in \mathscr{P}\left(E_{2}\right)$ and $B \in \mathscr{B}, b_{B, M}$ is the function from $E_{2}$ to $\mathscr{P}_{1}$ given by

$$
b_{B, M}(y)=\left\{\begin{array}{ll}
b_{B}(y) & \text { if } y \in M \\
\emptyset & \text { otherwise }
\end{array} \quad\left(y \in E_{2}\right)\right.
$$

and $b / M$ denotes the restriction of $b$ to $M$.

Proof - For any $N \in E_{2} / \mathscr{R}_{b}$ and $y \in N$,

$$
\begin{array}{rlr}
\left(\bigvee_{M \in E_{2} / \mathscr{B}_{b}} b_{B_{b / M}, M}\right)(y)= & b_{B_{b / N}}(y) & \text { (definition and property of } \left.b_{B, M}\right) \\
& =b / N(y) & \\
& =b(y) . & \text { (Proposition 7 applied to the ct-function } b / N \text { ) } \\
\text { (definition of restriction) }
\end{array}
$$

The locally ct-functions of Proposition 8 are the functions $b_{B, M}$.

We say that an elementary operator from $\mathscr{P}_{1}$ to $\mathscr{P}_{2}$ is locally c.t.i. iff its structuring function in $\mathscr{P}_{1}^{E_{2}}$ is a locally ct-function. For any $M \in \mathscr{P}_{2}$ and $B \in \mathscr{B}$, we denote by $\alpha_{B, M}$ (or ${ }_{B, M} \alpha$ ) the locally c.t.i. elementary operator which has the structuring function $b_{B, M}$.

\section{Elementary Operator Decomposition in terms of Locally c.t.i. Elementary Operators}

Theorem 9 - Any elementary operator $\delta$ (resp. $\varepsilon, \delta^{\text {a }}$ and $\left.\varepsilon^{\mathrm{a}}\right)$ of the class $\Delta\left(\mathscr{P}_{1}, \mathscr{P}_{2}\right)$ (resp. $\mathrm{E}\left(\mathscr{P}_{1}, \mathscr{P}_{2}\right), \Delta^{\mathrm{a}}\left(\mathscr{P}_{1}, \mathscr{P}_{2}\right)$ and $\left.\mathrm{E}^{\mathrm{a}}\left(\mathscr{P}_{1}, \mathscr{P}_{2}\right)\right)$ can be decomposed in terms of locally c.t.i. elementary operators of the same class and, if $b$ is its structuring function, the constructive decomposition is $\delta=\bigwedge_{M}{ }_{B_{b / M}{ }^{M}} \delta$ (respectively. $\varepsilon=\bigwedge_{M} \varepsilon_{B_{b / M}{ }^{M}}, \varepsilon^{\mathrm{a}}=\bigvee_{M} \varepsilon^{\mathrm{a}}{ }_{B_{b / M^{M}}}$ and $\left.\delta^{\mathrm{a}}=\bigvee_{M} B_{b / M^{M}} \delta^{\mathrm{a}}\right)$, where the union and intersection are taken over $E_{2} / \mathscr{\Re}_{b}$.

Proof - The result is a consequence of Propositions 1,2 and 8. The decomposition involves an union (resp. intersection) when the mapping $a \mapsto \alpha$ is an isomorphism (resp. a dual isomorphism). 
From Theorems 6 and 9, we see that any operator can be decomposed in terms of locally c.t.i. elementary operators.

Let $E$ be a non empty subset of $\mathbf{Z}^{2}$ and $B \in \mathscr{P}_{E . E}$. We now consider the example of decomposition of $\delta_{B}$, the ct-dilation by $B$ defined from $\mathscr{P}(E)$ to $\mathscr{P}(E)$. We know that $\delta_{B}(X)=(X \oplus B) \cap E$. The left kernel (as defined in Theorem 6) of $\delta_{B}$ is given by

$$
\text { - } \mathscr{G}_{\mathcal{G}}\left(\delta_{B}\right)(y)=\left\{X \in \mathscr{P}:\left(B^{\mathrm{t}}+y\right) \cap X \neq \emptyset\right\} \quad(y \in E) .
$$

For any $y \in E$, if $\left(B^{\mathrm{t}}+y\right) \cap E \neq \emptyset$, then $\cdot \mathscr{T}_{0}\left(\delta_{B}\right)(y) \neq \emptyset$ and the pairs $(a, b)$ of interest in the decomposition of $\delta_{B}$ are such that the $a(y)$ contain at least one point in $\left(B^{\mathrm{t}}+y\right) \cap E$; if $\left(B^{\mathrm{t}}+y\right) \cap E=\emptyset$, then $\cdot \mathscr{G}\left(\delta_{B}\right)(y)=\emptyset$ and by convention $a(y)=E$ and $b(y)=\emptyset$.

Let consider the following simple case where $B=\{p\}$ with $p \in E \oplus E^{\mathrm{t}}$ and let $Z=E \cap(E+p)$. The pairs $(a, b)$ of interest leading to the greatest interval functions reduce to only one defined by $a(y)=\{y-p\}$ and $b(y)=E$ if $y \in Z$, and $a(y)=E$ and $b(y)=\emptyset$ if $y \in E-Z$.

Hence, by Theorem $6, \delta_{\{p\}}=\varepsilon_{a} \wedge{ }_{b} \delta^{\text {a }}$ where $a$ and $b$ are the above ct-functions. We observe that even $\delta_{\{p\}}$ being a ct-dilation $\varepsilon_{a}$ is neither a ct-erosion nor a locally ct-erosion. Just ${ }_{b} \delta^{\mathrm{a}}$ is a locally ct-anti-dilation (with $M=Z$ and $B=E$ ). Nevertheless, by Theorem 9 , we can decompose $\varepsilon_{a}$ in terms of two locally ct-erosions: $\varepsilon_{a}=\varepsilon_{\{-p\}, Z} \wedge \varepsilon_{E, Z^{\circ}}$ Finally, we get the following decomposition of the ct-dilation $\delta_{\{p\}}$ in terms of locally cterosions and ct-anti-dilation:

$$
\delta_{\{p\}}=\left(\varepsilon_{\{-p\}, Z} \wedge \varepsilon_{E, Z^{c}}\right) \wedge_{E, Z} \delta^{\mathrm{a}} .
$$

\section{Acknowledgements}

The authors are grateful to Professor J.Serra who has suggested the present work during the previous workshop at Barcelona in May 1993 as well as the names of "conditional translation invariance" and "ct-operator".

This work has been supported by FAPESP (Fundação de Amparo à Pesquisa do Estado de São Paulo) under contract 91/3532-2.

\section{References}

Achache, A.: 1982, "Galois Connexion of fuzzy subset”. Fuzzy Sets and Systems, Vol. 8, pp. 215-218.

Banon, G. J.F. and Barrera, J.: 1990, "Set mapping decomposition by Mathematical Morphology”. In R. M. Haralick ed., Mathematical Morphology: Theory and Hardware. To appear.

Banon, G. J.F. and Barrera, J.: 1991, "Minimal representations for translation-invariant set mappings by Mathematical Morphology". SIAM J. Appl. Math. Vol. 51, pp. 1782-1798.

Banon, G. J. F. and Barrera, J.: 1993a, "Decomposition of mappings between complete lattices by Mathematical Morphology". Signal Processing Vol. 30, pp. 299-327.

Banon, G. J.F. and Barrera, J.: 1993b, “A decomposition theorem in Mathematical Morphology”. Proceedings of the International Worshop on Mathematical Morphology and its Application to Signal Processing, Barcelona, Spain, pp. 234-238.

Birkhoff, G.: 1967, Lattice theory. $3^{\text {rd }}$ ed., American Mathematical Society, Providence, Rhode Island.

Hadwiger, H.: 1950, "Minkowskische Addition und Subtraktion beleibiger Punktmengen und die Theoreme von Erhard Schmidt". Math. Zeitschrift. Vol. 53, pp. 210-218.

Serra, J.P.F. (edited by): 1988, Image Analysis and Mathematical Morphology. Volume 2: Theoretical Advances. Academic Press, London, 411 p.. 